\title{
Diseño de un prototipo de robot con geometría Rocker-Bogie
} (Design of a Robot Prototype with Rocker Geometry-Bogie)

\author{
Christian Montaleza1', Xavier Mayorga², Jimmy Gallegos ${ }^{3}$ y Rogelio León 4 .
}

\begin{abstract}
Resumen
En la presente investigación se diseña y valida una geometría de suspensión tipo Rocker-Bogie basados en el análisis de grados de libertad para su futura implementación dentro del sector de la industria de exploración espacial. Ello tiene como finalidad principal la recolección, transporte de basura y desperdicios a sectores estratégicos dentro de las instalaciones, sorteando obstáculos de diferente nivel, aunque también podría aplicarse en el área industrial, agrícola y minera, en las que se requiera un sistema robusto y estable. Se parte estableciendo un factor de seguridad determinado a través de coeficientes que involucren seguridad y economía. Luego, se realiza el cálculo de las dimensiones y fuerzas a soportar por los elementos estructurales conocidos como Rocker y Bogie que son los encargados de transmitir el soporte al chasis, además de brindar soporte a las conexiones para los componentes de los ejes y motores para las ruedas. Del diseño y cálculo de estos elementos depende que el prototipo de robot sea capaz de movilizarse a través de campos irregulares sorteando obstáculos como: anomalías en la superficie, baches, rocas y bordillos. Para la validación del diseño del prototipo se realiza un análisis estático de los elementos estructurales (Rocker y Bogie) por medio del parámetro de Von Misses, obteniendo un nivel de seguridad elevado antes de la ruptura. Posteriormente, se ejecuta la simulación del prototipo en base a una pista creada en el software analizando parámetros de velocidad, aceleración y desplazamientos en los ejes X y Y. Finalmente, se comparan los resultados obtenidos de la simulación basándose, principalmente, en altura y longitud máxima entre eje frontal y posterior, siendo crucial el ángulo máximo de inclinación permisible.
\end{abstract}

\section{Palabras clave}

Rocker-Bogie, diseño, geometría, robot, simulación.

\begin{abstract}
In this research a Rocker-Bogie type suspension geometry is designed and validated based on the analysis of the degrees of freedom, for its future implementation within the space exploration industry sector, having as main purpose the collection and transportation of garbage and waste to strategic sectors within the facilities, avoiding obstacles of different levels, although it could also be applied in the industrial, agricultural and mining areas, where a robust and stable system is required. We start by establishing a safety factor, determined through coefficients that involve safety and economy, then we calculate the dimensions and forces to be supported by the structural elements known as Rocker and Bogie, which are responsible for transmitting the support to the chassis as well as providing support to the connections for the axle components and motors for the wheels. The design and calculation of these elements, depends on the robot prototype being able to move through irregular fields avoiding obstacles such as: surface anomalies, potholes, rocks and curbs. For the validation of the prototype design, a static analysis of the structural elements (Rocker and Bogie) is performed by means of the Von Misses parameter, obtaining a high level of safety before rupture. Subsequently, the simulation of the prototype is executed based on a track created in the software, analyzing parameters of speed, acceleration and displacements in the axes $X$, $Y$. Finally, the results obtained from the simulation are compared based mainly on height and maximum length between front and rear axles, being crucial the maximum permissible angle of inclination.
\end{abstract}

\section{Keywords}

Rocker-Bogie, design, geometry, robot, simulation.

\footnotetext{
$1 \quad$ Universidad de Jaén. Jaén, España. [cimg0001@red.ujaen.es, https://orcid.org/0000-0003-1101-9527]

2 Universidad Europea de Madrid. Madrid, España. [21958869@live.uem.es, https://orcid.org/0000-0001-9840-924X]

3 Universidad de Jaén. Jaén, España. [jwga0001@red.ujaen.es, https://orcid.org/0000-0003-3792-0620]

$4 \quad$ Universidad de Jaén. Jaén, España. [rslj0001@red.ujaen.es, https://orcid.org/0000-0003-2142-3769]
} 


\section{Introducción}

Los esfuerzos de la humanidad por estudiar el espacio desde el punto de vista científico y económico se denomina exploración espacial (Hu et al., 2020). La exploración espacial se logra utilizando robots en un ambiente abarrotado de obstáculos con el fin de recabar datos del entorno (Gul et al., 2021). A lo largo de la historia se han desarrollado múltiples exploraciones con diferentes robots (Bogue, R., 2012). Uno de estos robots es el Rocker-Bogie, más conocido como Rover utilizado por la NASA (Karras et al., 2017; Tashtoush et al., 2020).

El propósito de los robots es ayudar, colaborar o incluso reemplazar a los seres humanos en actividades muy detalladas, repetitivas, complicadas, difíciles o peligrosas (Gonzales y lagnemma, 2017) a través de complejos movimientos con velocidad, fuerza y alto grado de precisión de manera segura y económica (Conte, 2013). Esto se logra con la integración de sistemas mecánicos, eléctricos, electrónicos y computacionales dentro de un mismo dispositivo programable (Xu y Wang, 2021). El prototipo o geometría dentro de la simulación es el primer paso que se realiza (Misha et al., 2020).

En la figura 1 se observa la evolución de los robots de exploración en cuanto a los últimos 50 años en los que se ha dirigido un esfuerzo sustancial en la investigación hacia el desarrollo de conceptos y prototipos:

- El primer prototipo de exploración lunar fue enviado por la Unión Soviética para la recolección de información del terreno con transmisión de fotos. Este prototipo llamado Lunokhod recorrió unos 10.5 km durante su viaje de 11 meses y tenía un diseño muy precario sin la capacidad de hacer girar las ruedas, por lo que el giro se lograba acelerando la velocidad de rotación de las ruedas en los trenes derechos e izquierdos.

- En 1996 la Nasa diseñó a Rover Sojourner, este robot se asemejaba a un horno microondas con un peso de $10 \mathrm{~kg}$, este sistema le permitía superar los obstáculos superiores hasta 1.5 veces el diámetro de la rueda (Eisen et al., 1998).

- Por otra parte, Rocky 7 presentaba un diseño similar al del Sojourner, se diseñó con un brazo robótico unido al cuerpo para facilitar las investigaciones y su sistema de dirección cumplía con el tipo Ackerman, disminuyendo complejidad, pero comprometiendo su maniobrabilidad.

- Siguiendo este recuento se encuentra Rover SRR, este robot fue diseñado para recoger muestras del suelo y cuenta con un sistema de suspensión activo debido a su ángulo de viraje, en superficies inclinadas la suspensión activa puede mantener el cuerpo principal en ángulo horizontal.

- El robot Nano Rover fue diseñado para la exploración de pequeños cuerpos celestes como los cometas y asteroides. Las pequeñas dimensiones son la ventaja de este robot, consta con 4 ruedas de $6 \mathrm{~cm}$ de diámetro, con capacidad de operación en ambos lados, no existe inconveniente en caso de volcamiento (Smith y Saaj, 2009).

- Shrimp Rover fue diseñado con 6 ruedas y un sistema de 4 barras específicas para poder sortear obstáculos de hasta 2 veces el tamaño de sus ruedas sin perder estabilidad. Contiene un Bogie paralelogramo que equilibra las fuerzas de reacción en cada rueda y cuenta con una rueda trasera conectada directamente al cuerpo principal que es accionada por un motor para aumentar la capacidad de escalar (Meghdari et al., 2005).

- Finalmente, Mars Exploration Rover (MER), en 2004 se incluyó el envió de 2 robots exploradores idénticos llamados Spirit y Oportunity, su función principal constaba en la bús- 
queda de la presencia de agua en rocas y suelos, los robots median $1.6 \mathrm{~m}$ de largo, peso de 150 kg y cuenta con el sistema de suspensión llamado Rocker-Bogie (Tunstel, 2007).

- Uno de los últimos robots enviados a exploración en 2012 es el llamado Curiosity, pesa $899 \mathrm{~kg}$ con $75 \mathrm{~kg}$ de instrumentos científicos, con una longitud de $3 \mathrm{~m}$, ancho de $2.8 \mathrm{~m}$, altura de $2.2 \mathrm{~m}$ y un brazo robótico de $2.1 \mathrm{~m}$. Su velocidad máxima es de $0.144 \mathrm{~km} / \mathrm{h}$ al tener un motor eléctrico independiente en cada una de las 6 ruedas que posee (Toupet et al., 2018).

Figura 1. Robots Rover de exploración: a) Lunokhod (Sanguino, 2017). b) Sojurner and Oportunity (Sanguino, 2017). c) Rocky 7 (Volpe et al., 1996).

d) Nano (Smith, y Saaj, 2009). e) Shripm (Yang et al., 2017). f) Curiosity (Kornfeld et al., 2014)
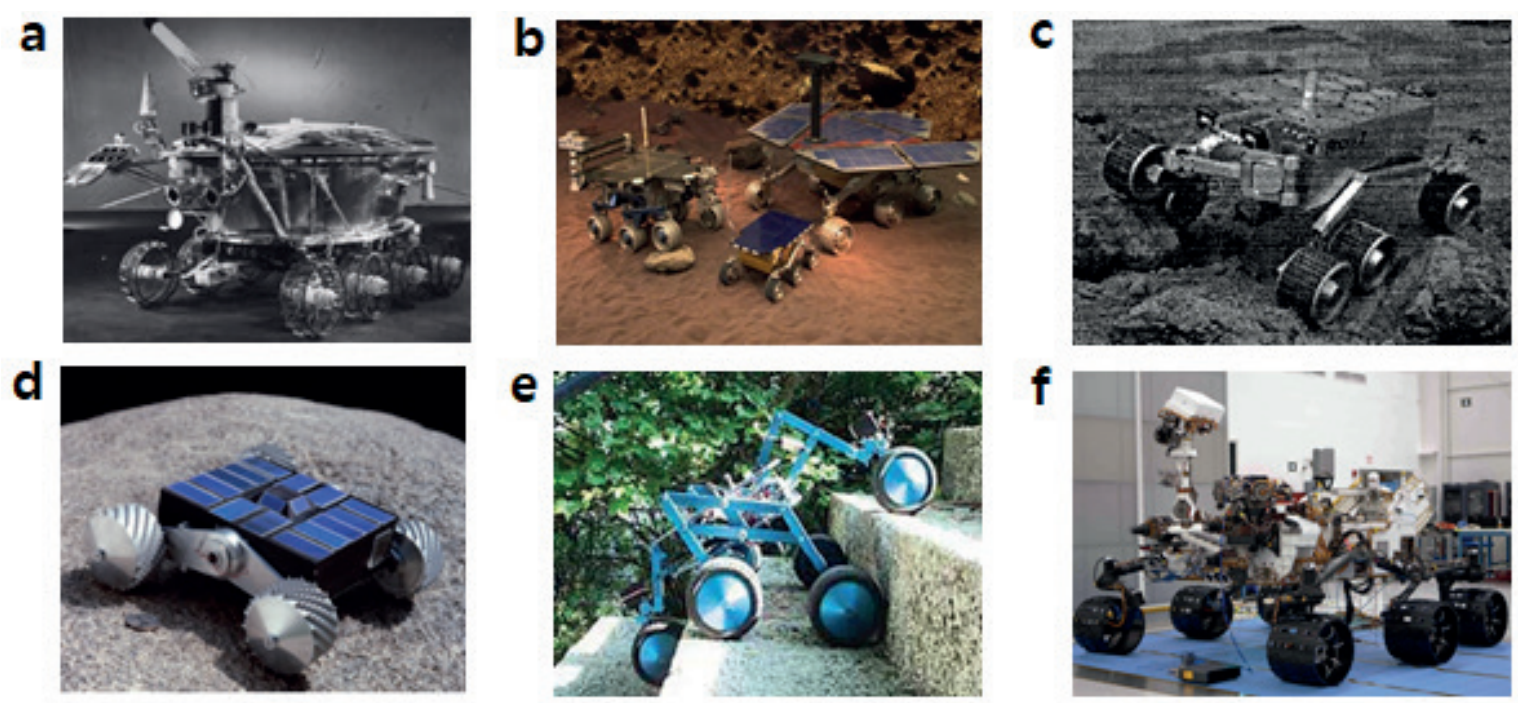

Sin duda, los robots Rover son mayormente utilizados en exploraciones espaciales debido a su extenso patrimonio (Chinchkar et al., 2017), ya que lo que se busca en el diseño es una geometría tan simple como sea posible (Zheng et al., 2018). En la mayoría de los casos no se requeriría sistema de suspensión, pero no se puede evitar, ya que el sistema de suspensión Rocker-Bogie permite al robot superar obstáculos (Kim et al., 2012). En lo viable, se trata de diseñar una geometría sencilla, reduciendo el coste computacional en la ejecución de la simulación (Cabrera et al., 2021) al incorporar, no solo la geometría del prototipo, sino datos de entrada o contorno con el fin de anticiparse al proceso real, validarlo y, de ser necesario, rediseñarlo para obtener la mejor configuración (Hu et al., 2019).

Esta investigación pretende contribuir primero: con un diseño de geometría relativamente simple y ligera, permitiendo que la movilidad se guarde dentro del espacio limitado disponible. Segundo: implementar una configuración móvil segura para que pueda explorar la superficie. Tercero: que sea capaz de sortear obstáculos de diferente nivel a lo largo de la superficie, manteniendo la estabilidad.

La investigación se compone de una sección de metodología en la que se establecen datos de partida, factor de seguridad y se realizan los cálculos y modelados de piezas estructurales llamadas Rocker y Bogie de dos conceptos diferentes de diseño. Para la validación se realiza un análisis estático en el cual, a través del parámetro de Von Misses, se determina si es capaz 
de soportar diferentes esfuerzos. La sección de resultados muestra un análisis dinámico en el que se determina si el prototipo es capaz de sortear obstáculos de diferente nivel. Luego, en la sección de discusión se realiza una comparativa entre los prototipos y estudios ya existentes. Finalmente, en la sección de conclusiones y recomendaciones, aparte de hacer un análisis crítico, se aporta con futuras líneas de investigación que continúen con un análisis más profundo de los robots Rover, como la programación de controladores electrónicos de estabilidad.

\section{Metodología}

Para el diseño del prototipo de suspensión se deben establecer datos de partida como $\boldsymbol{m}$ (masa total de diseño), $\boldsymbol{R}$ (radio de la rueda) y $\boldsymbol{\theta}_{\text {máx }} \boldsymbol{d}$ (ángulo máximo de inclinación), además de establecer un factor de seguridad $\left(n_{s}\right)$ que permita evaluar el coeficiente admisible de seguridad como el productor de dos factores que involucran aquellos determinantes en su valoración (Musto, 2010). La ecuación 1 es tomada del método de A. G Pugsley para determinar dicho factor (Elishakoff, 2017).

$n_{s}=n_{s x}^{*} n_{s y}$

Donde $\boldsymbol{n}_{\boldsymbol{s} \boldsymbol{x}}$ es el factor de seguridad que involucra aspectos de control y calidad. Y $\boldsymbol{n}_{\boldsymbol{s} \boldsymbol{y}}$ es el factor de seguridad que involucra aspectos de seguridad y economía.

Se busca que el factor de seguridad de diseño sea mayor que 1 para estar dentro de la normativa de diseño y se pueda continuar con el cálculo de los componentes del sistema de suspensión Rocker-Bogie.

\subsection{Primer prototipo}

En la figura 2 se establecen los grados de libertad que corresponden al mecanismo. Es decir, el número de reacciones de la estructura, obteniendo dicho valor con la ecuación 2.

Figura 2. Grados de libertad para el diseño de la geometría Rocker-Bogie

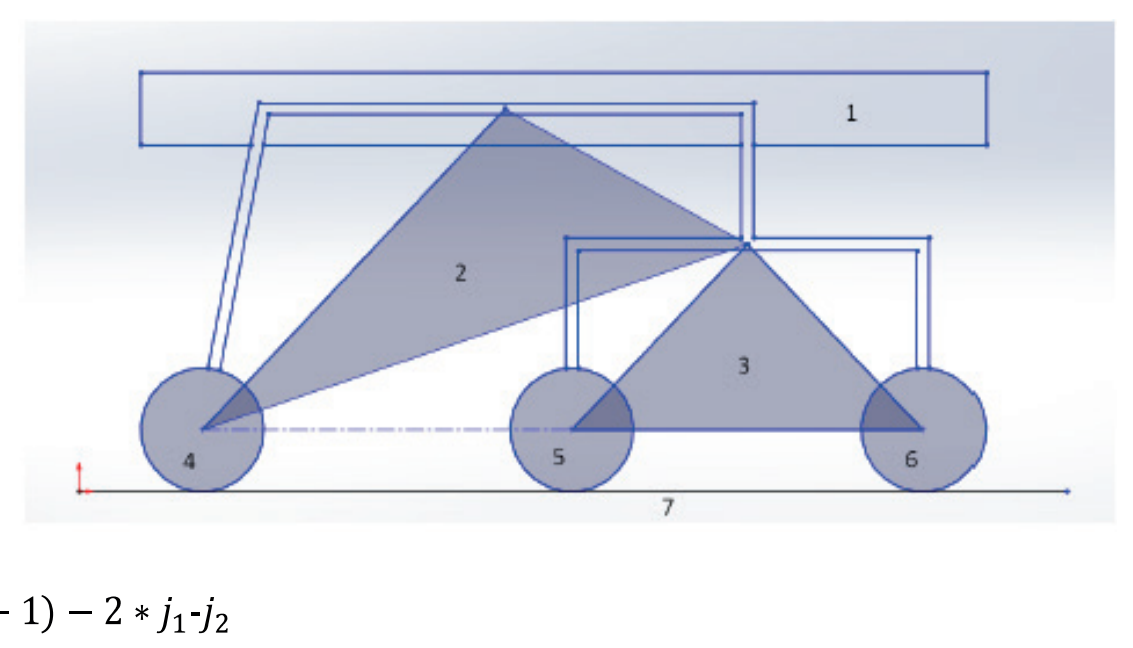

Donde $\boldsymbol{m}_{\boldsymbol{g} \boldsymbol{l}}$ son los grados de libertad, $\boldsymbol{n}$ el número de elementos del mecanismo, $\boldsymbol{j}_{\mathbf{1}}$ el número de uniones de un grado de libertad, $\boldsymbol{j}_{2}$ el número de uniones de dos grados de libertad. 
El peso total a soportar por la estructura (W) se determina con la ecuación 3. La figura 3 representa el diagrama de cuerpo libre sobre la estructura que forma el Rover, donde $\boldsymbol{h} \mathbf{l}$ es la distancia desde el eje de la rueda hasta el eslabón del Bogie, $\mathbf{h} \mathbf{2}$ es la altura desde el centro de las ruedas hasta el eslabón Rocker, $\boldsymbol{F}$,, $\boldsymbol{F} \mathbf{2}$ y $\mathbf{F 3}$ serán consideradas como las fuerzas de reacción producidas por la superficie al contacto con las ruedas.

Figura 3. Diagrama de cuerpo libre

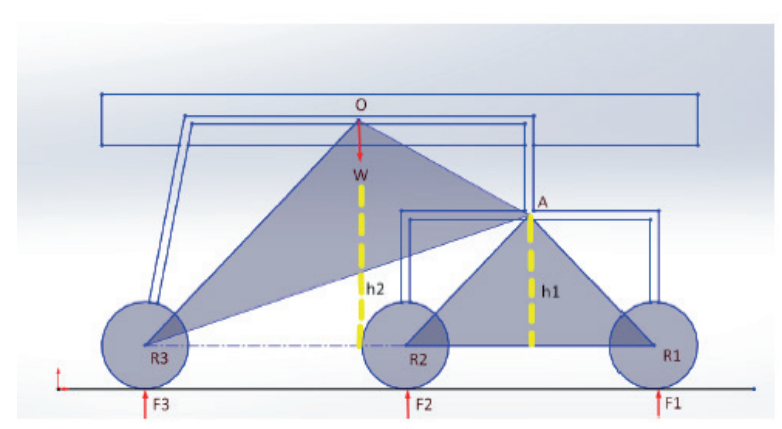

$W=m^{*} g$

Por medio de la sumatoria de fuerzas en A (ecuación 4) y en 0 (ecuación 5) se calcula las fuerzas de reacción en cada una de las conexiones de los eslabones Rocker-Bogie en los ejes de las ruedas.

$\Sigma M_{A}=0$

$\Sigma M_{O}=0$

La ecuación 6 representa la distancia entre ruedas. Para lo cual se debe establecer el diámetro de la rueda y la longitud total.

$L_{r}=\frac{L_{t}-D_{r}}{2}$

Para determinar la altura de los eslabones Rocker-Bogie se realiza un diagrama mediante la separación de triángulos rectángulos, tal como se muestra en la figura 4. Las ecuaciones 7, 8, 9 y 10 representan a las funciones trigonométricas necesarias para calcular dicha altura.

Figura 4. Diagrama de altura de los eslabones: a) eslabón Bogie, b) eslabón Rocker.

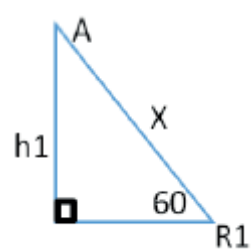

a)

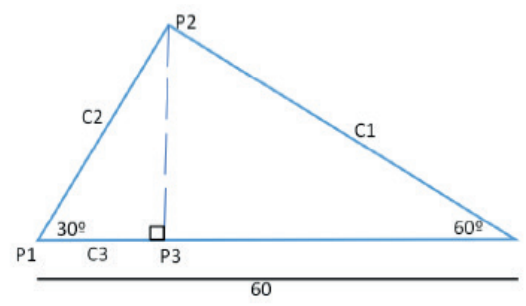

b) 
$\cos (60)=\frac{15}{x}$

$\sin (30)=\frac{C_{1}}{60}$

$\cos (30)=\frac{C_{2}}{60}$

$\cos (30)=\frac{C_{3}}{C_{2}}$

La ecuación 11 representa la altura Rocker denominada distancia P2P3.

$\sin (30)=\frac{P 2 P 3}{C_{2}}$

La figura 5 muestra el bosquejo de la primera estructura calculada para el diseño de la geometría Rocker-Bogie.

Figura 5. Bosquejo principal geometría Rocker-Bogie

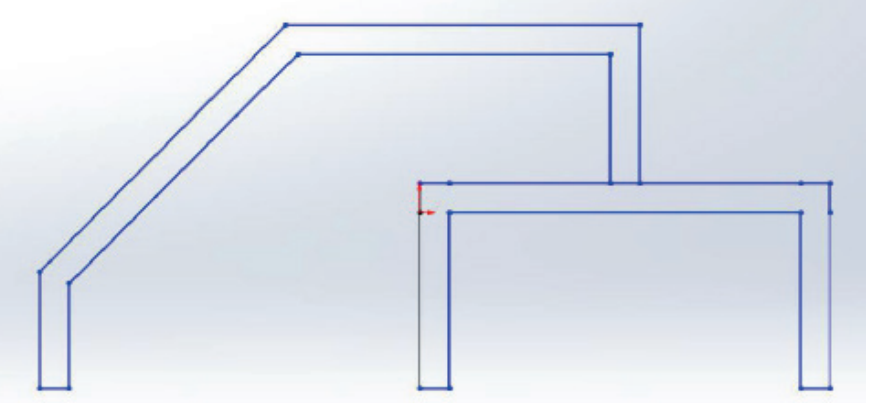

La carga en rueda $\left(\boldsymbol{C}_{\boldsymbol{r}}\right)$ se determina con la ecuación 12 a través de la carga total $\left(\boldsymbol{C}_{\text {Total }}\right.$ ) y el número de ruedas del prototipo ( \# ruedas). Con este parámetro determinado y con la ecuación 13 se calcula el torque $(\boldsymbol{T})$ necesario para iniciar el movimiento, multiplicando la fuerza (F) por la distancia $(\boldsymbol{d})$.

$C_{r}=\frac{C_{\text {Total }}}{\# \text { ruedas }}$

$T=F^{*} d$

\subsection{Segundo prototipo}

Repetimos el proceso anteriormente detallado para diseñar un nuevo concepto de geometría Rocker-Bogie, en el cual se consideró un diseño más geométrico como se muestra en la figura 6, 
buscando incrementar el ángulo de soporte y distribución de cargas soportadas por las ruedas. Las ecuaciones 14 y 15 permiten determinar la altura de los nuevos eslabones Rocker y Bogie.

Figura 6. Diseño y bosquejo de la segunda geometría Rocker-Bogie
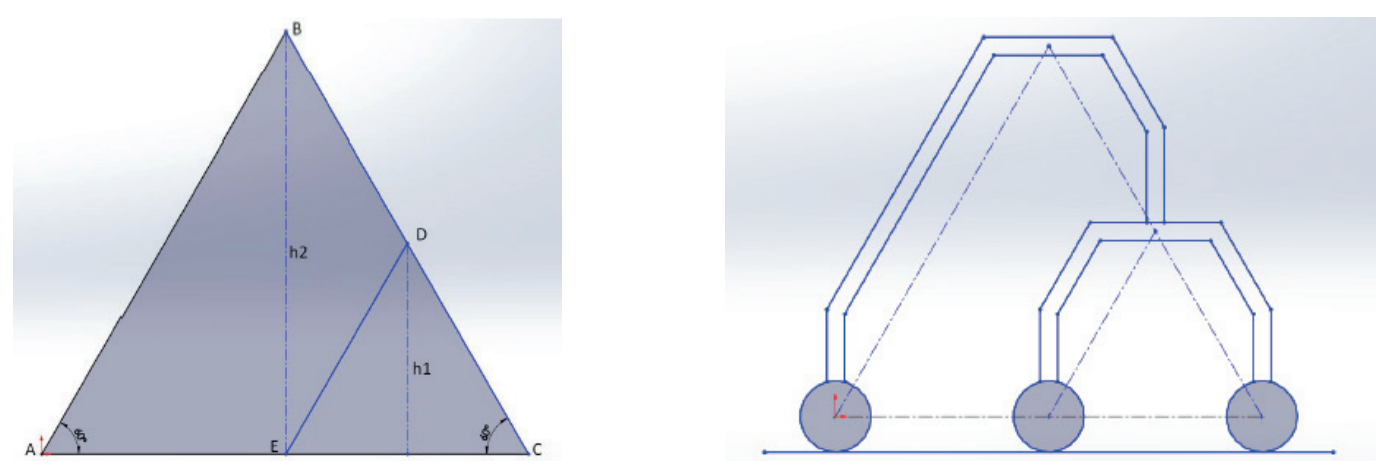

$\triangle E D C$

$h_{1}=\sin (60) *(30)$

$\triangle B E C$

$h_{2}=\sin (60) *(60)$

\subsection{Pendiente}

Con los valores establecidos en el diseño de las geometrías se determinan los valores del centro de gravedad de las suspensiones denominadas XCGL y ZCG. Se reemplazan los valores en la ecuación 16 que nos permiten calcular el ángulo de inclinación que podrá soportar el diseño. Ver la figura 7.

Figura 7. Centro de masa
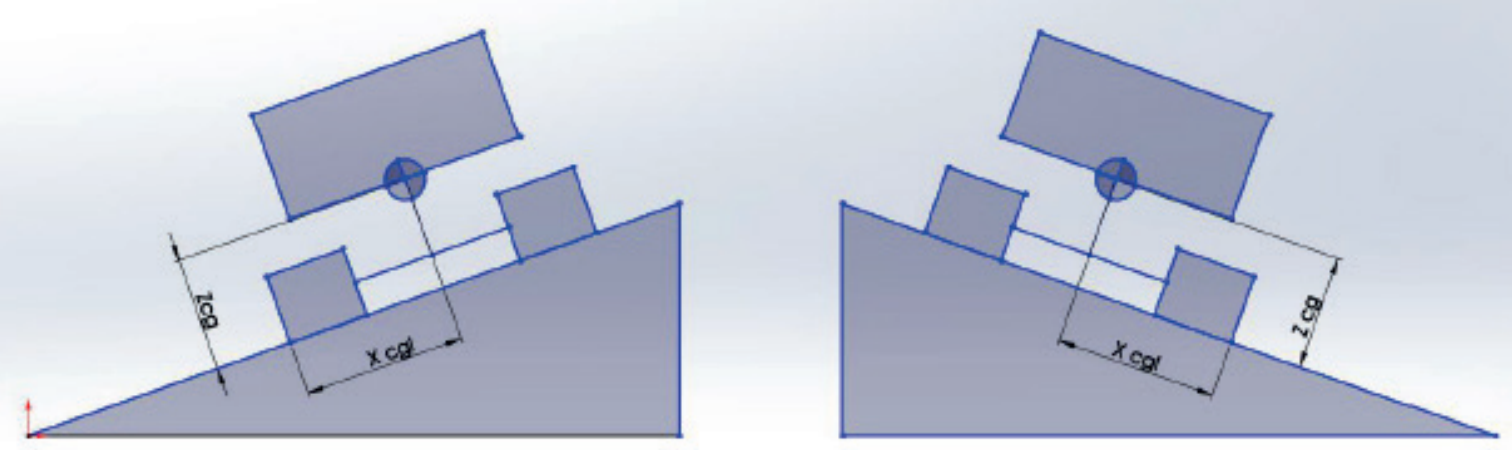

$\theta_{\text {máx }}=\min \left(\tan ^{-1}\left(\frac{X_{C G L}}{Z_{C G}}\right) ; \tan ^{-1}\left(\frac{X_{C G R}}{Z_{C G}}\right)\right)$

Se busca establecer un ángulo cercano a 30․ Por lo tanto, el diseño, partiendo desde el factor de seguridad, cumple adecuadamente con la geometría diseñada. 


\subsection{Tracción rueda}

Para el cálculo de la tracción ejercida por la rueda del prototipo se emplea el coeficiente requerido para la interacción entre material de la rueda en contacto con la superficie en donde se desliza. Se especifica que el coeficiente dinámico es 0.6 correspondiente al rozamiento caucho/madera y materiales del prototipo. Además, con una aceleración promedio de $0.02 \mathrm{~m} / \mathrm{s}^{2}$, obteniendo el valor de la fuerza empleada a partir de la ecuación 17 y 18.

$\Sigma M_{y}=0$

$\Sigma M_{z}=\mathrm{m} * \mathrm{a}$

\subsection{Diseño CAD}

En la figura 8 se observa el modelo CAD del Rover. La parte (a) representa el ensamble del primer modelado del sistema Rocker-Bogie que compone el Rover, destacando la ubicación del chasis con mayor cercanía al suelo. La parte (b) representa el ensamble del segundo modelado del sistema Rocker-Bogie que compone el Rover, destacando el cuerpo central, el cual debe mantener el equilibrio al pasar cada obstáculo.

Figura 8. Modelo CAD Rover

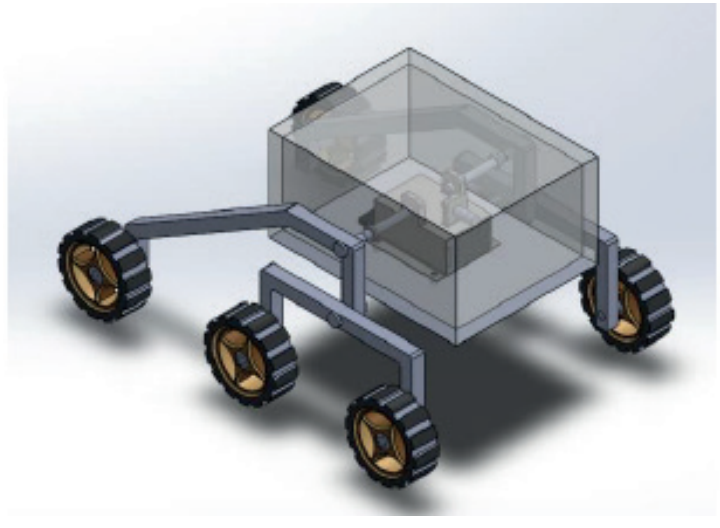

a) Primer prototipo

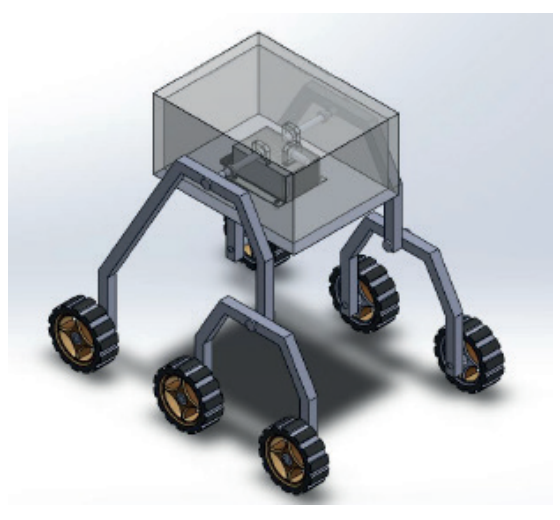

b) Segundo prototipo

\subsection{Validación}

La figura 9 muestra el análisis estático de los eslabones Rocker-Bogie, obteniendo un valor de Von Misses de 1.373e7 N/m2 para el eslabón Rocker, lo que permite interpretar que con el paso del tiempo lo primero que podría fallar son las conexiones, mientras que el eslabón Bogie tiene un valor de Von Misses de 2.283e7 N/m2, presentando un desgaste en los centros de conexión con cada uno de los ejes. 
Figura 9. Análisis estático

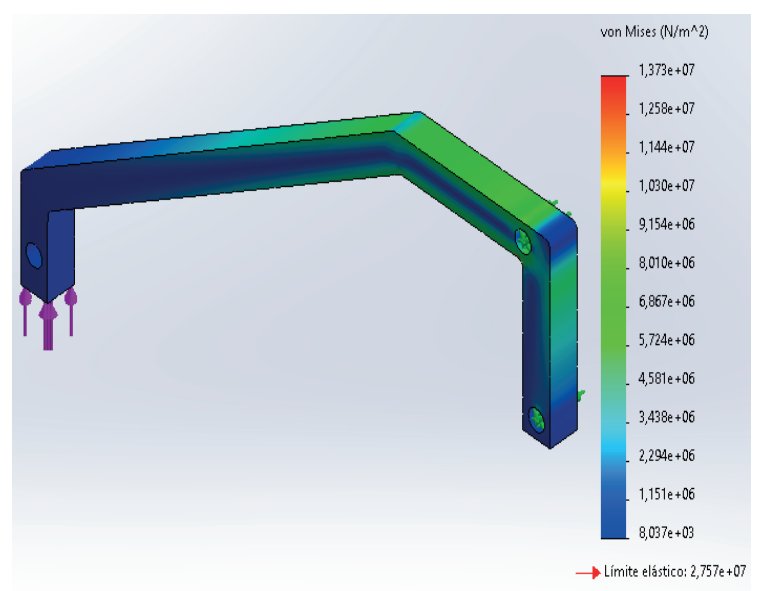

a) Eslabón Rocker

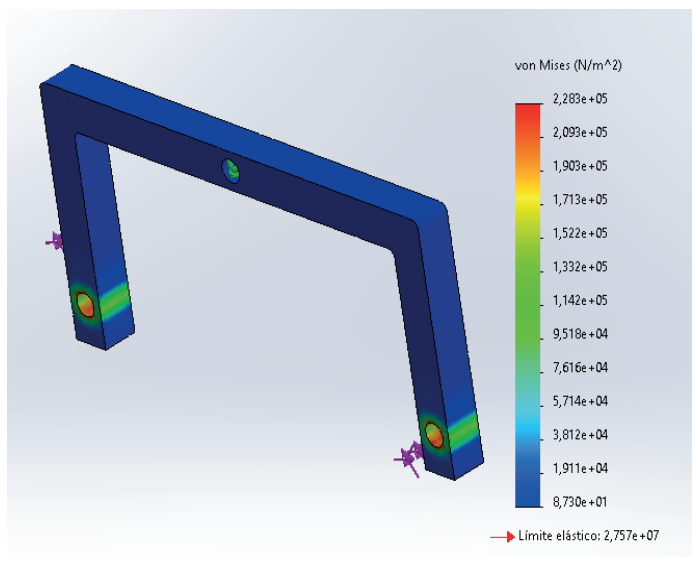

b) Eslabón Bogie

La figura 10 establece el valor máximo y mínimo de los factores de seguridad de los eslabones Rocker. Además, se comprueba que cumplen con el factor de diseño establecido. El valor entregado por el software para el factor de seguridad es de 2.01 para el eslabón Rocker del primer diseño parte (a) y de 3.83 para el segundo diseño parte (b), siendo superior al establecido en los cálculos previamente. Por lo cual, el diseño es adecuado.

Figura 10. Factor de seguridad eslabón Rocker

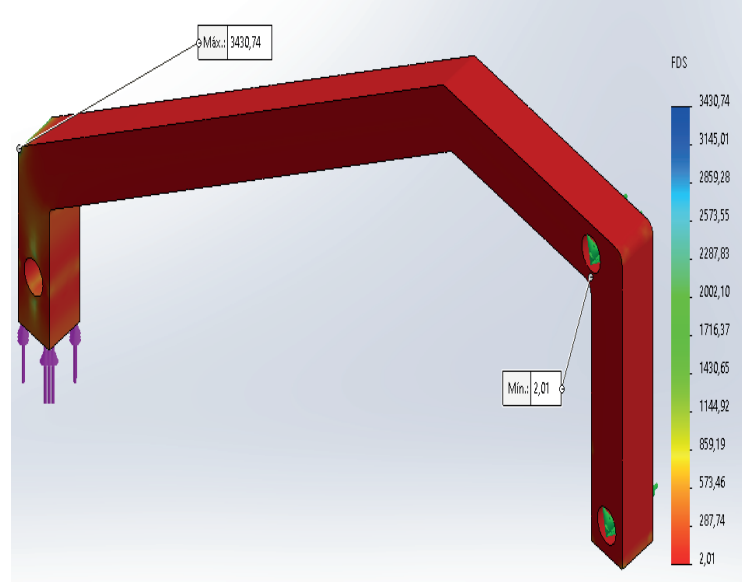

a) Primer diseño

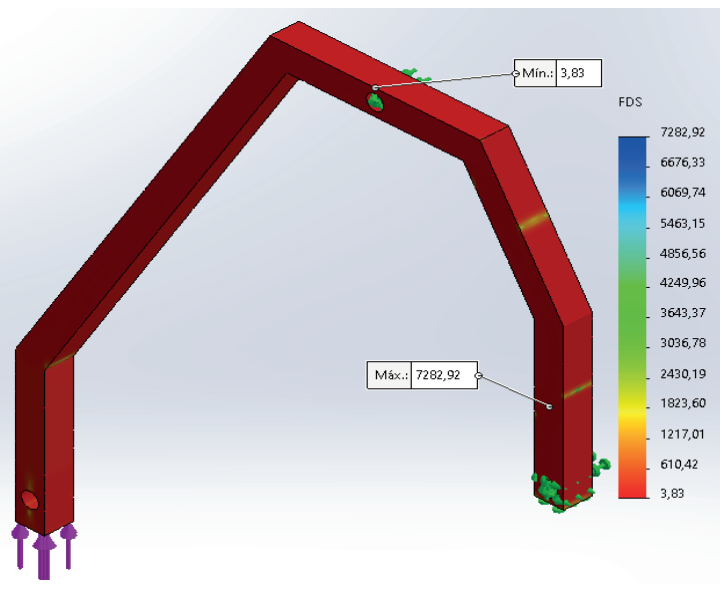

b) Segundo diseño

La figura 11 muestra que los valores para el factor de seguridad de los eslabones Bogie también son elevados a los calculados previamente. Por lo cual, el diseño también es adecuado para su implementación. 
Figura 11. Factor de seguridad eslabón Bogie

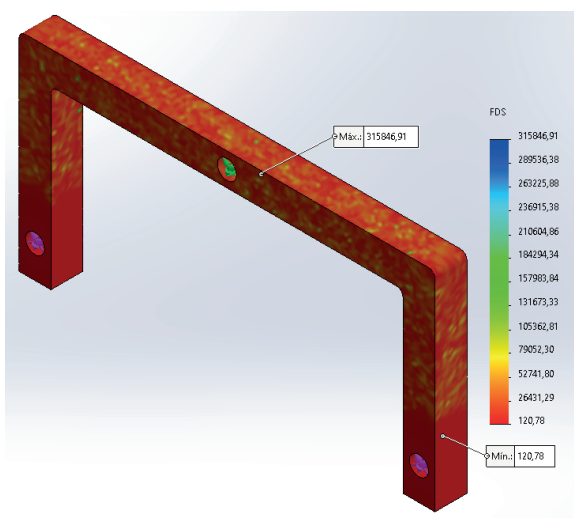

a) Primer diseño

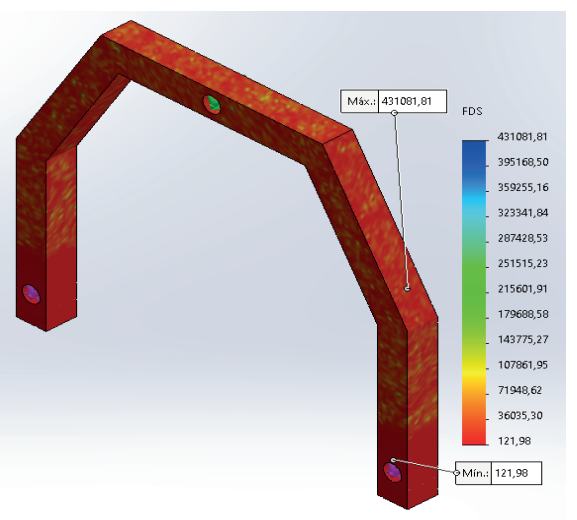

b) Segundo diseño

\section{Resultados}

Se realiza un análisis dinámico para el estudio del movimiento analizando parámetros específicos como: velocidad, aceleración y desplazamientos en los ejes X y Y. Determinando, de esta manera, la velocidad y aceleración máxima y mínima alcanzada por el Rover en condiciones normales y al atravesar obstáculos en la pista diseñada. En la figura 12, 13 y 14 observamos el comportamiento del primer prototipo de geometría diseñado. Este consta de eslabones que mantienen el chasis más cerca del suelo.

Figura 12. Distancia entre ejes primer prototipo

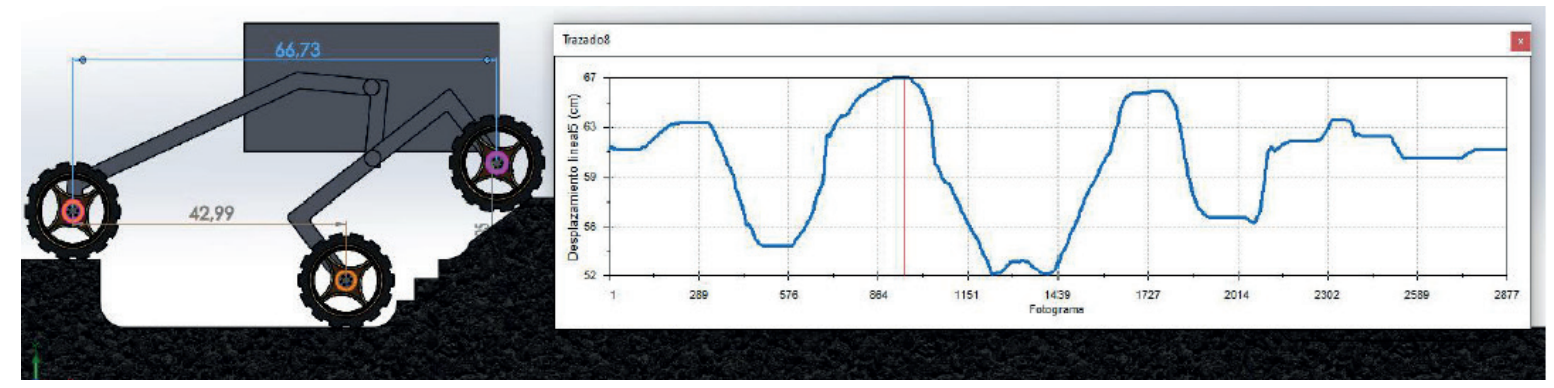

Figura 13. Diagrama de velocidades primer prototipo

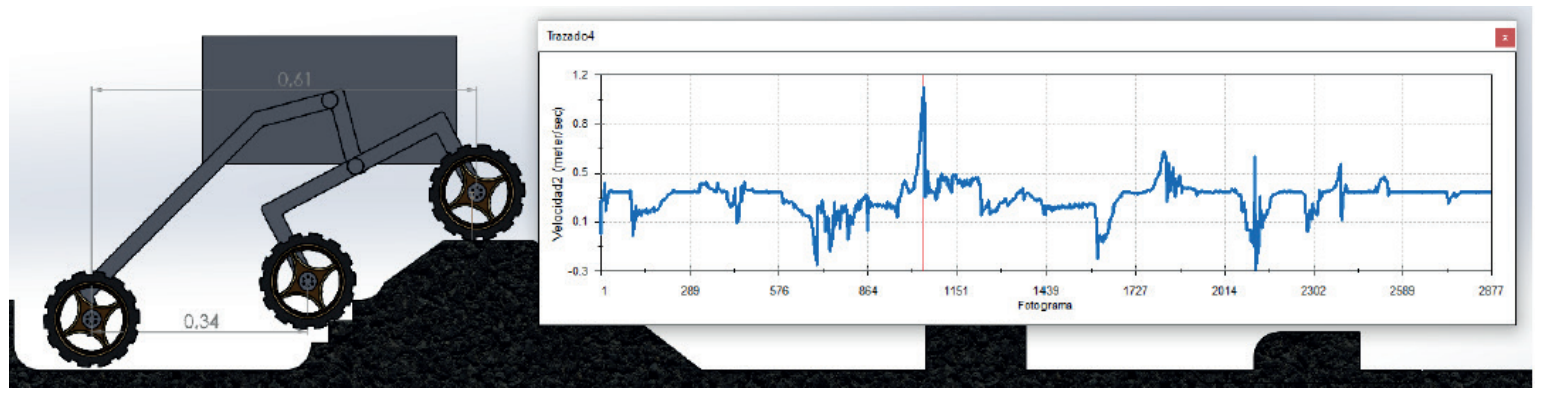


Figura 14. Diagrama de la fuerza torsional primer prototipo

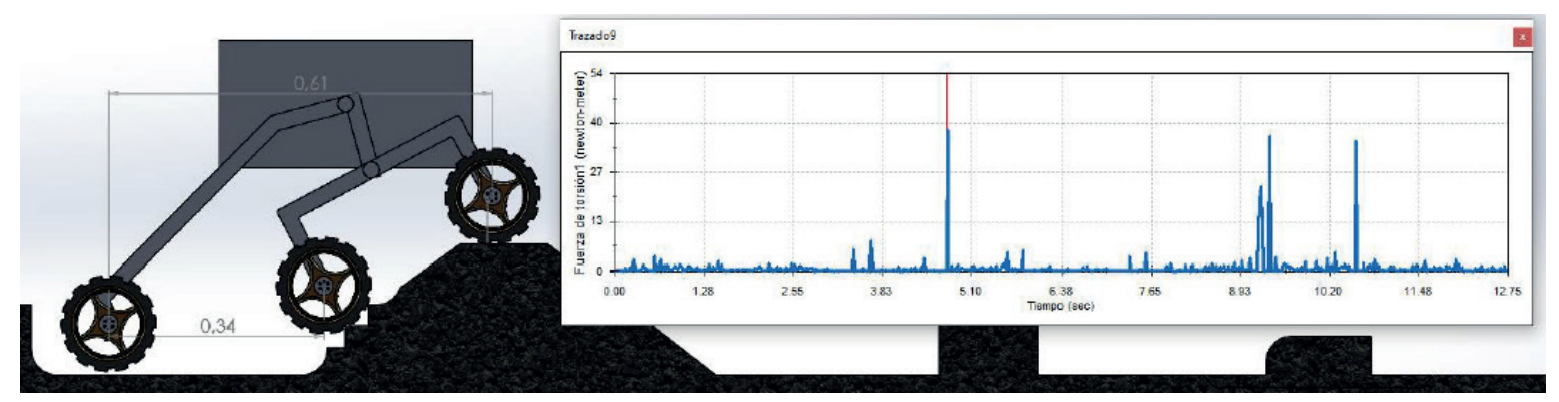

En la figura 15, 16 y 17 observamos el análisis dinámico del segundo prototipo de geometría diseñado.

Figura 15. Desplazamiento Rover segundo prototipo

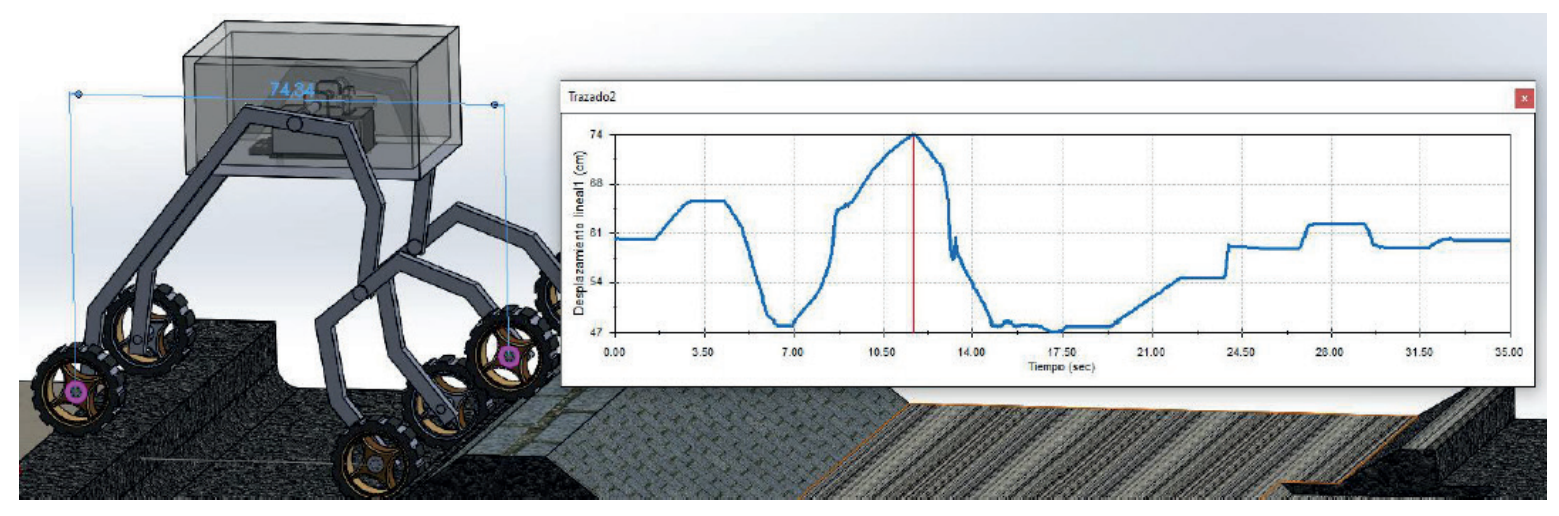

Figura 16. Diagrama de altura segundo prototipo

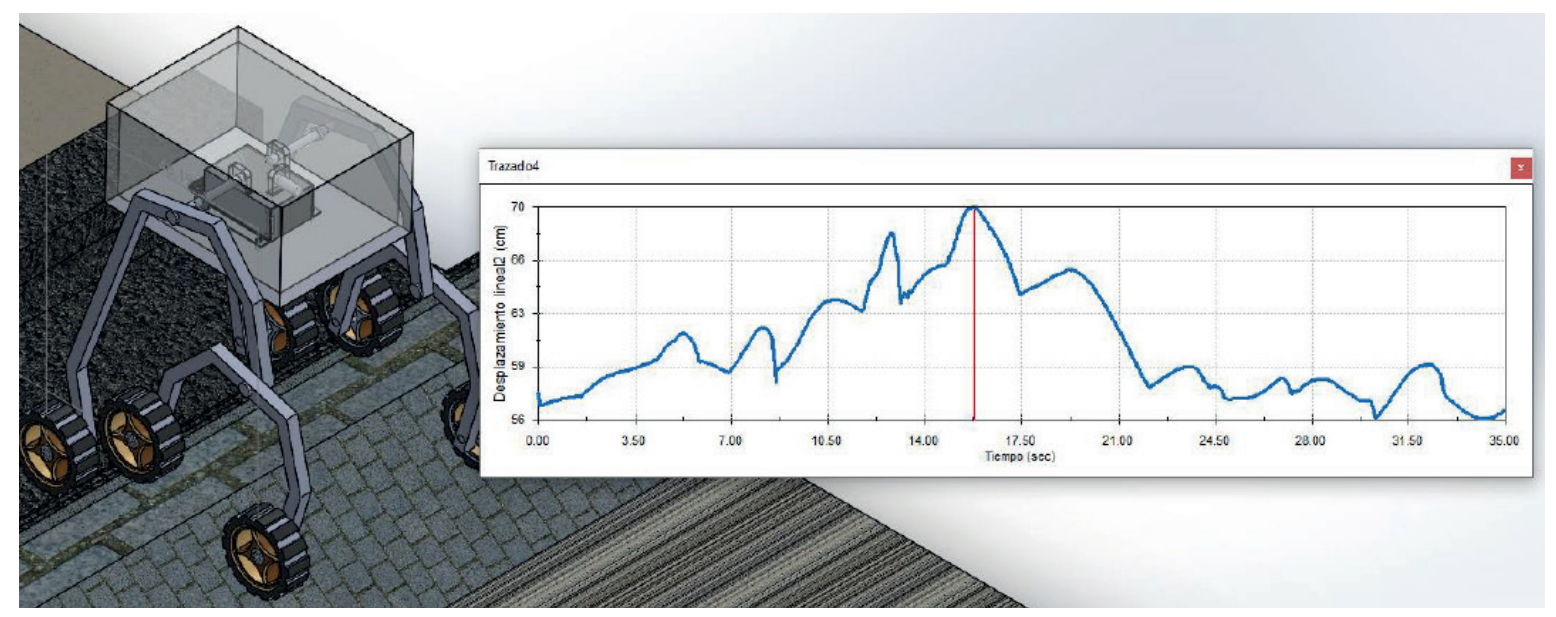


Figura 17. Diagrama de velocidades segundo prototipo

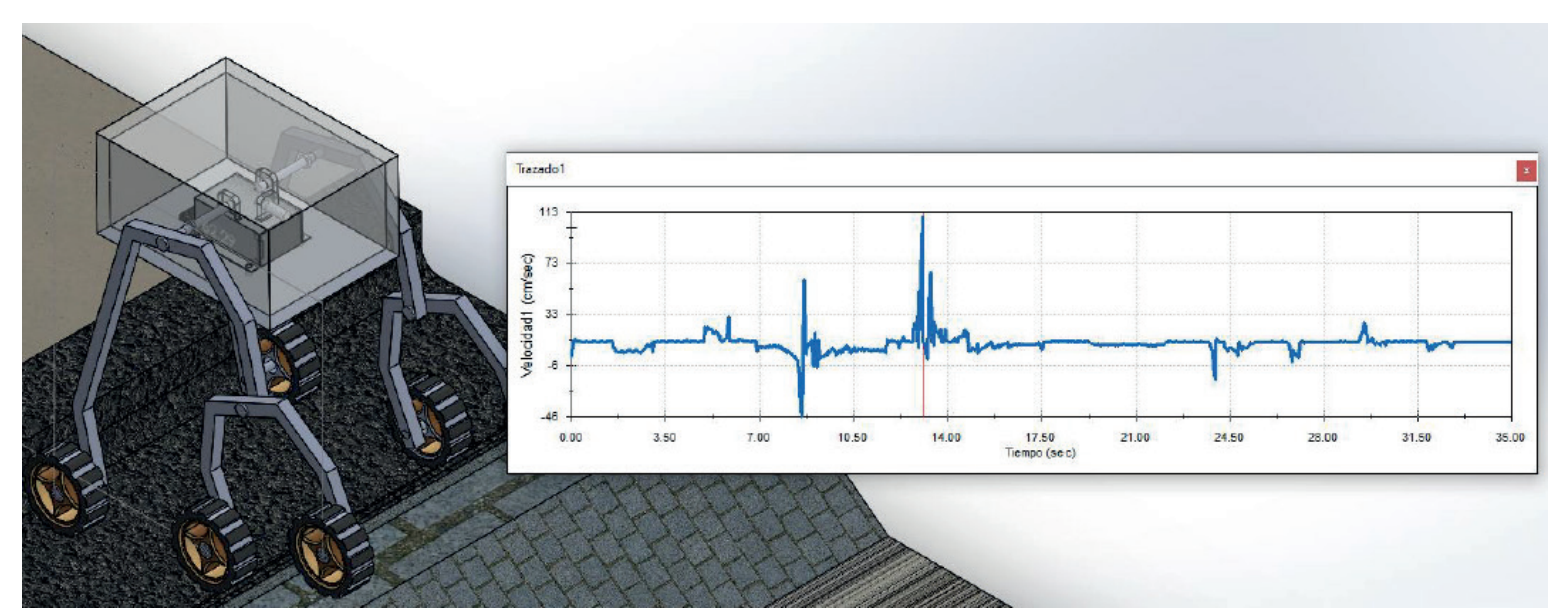

\section{Discusión}

En la tabla 1 observamos una comparativa entre los factores principales analizados en cada uno de los mecanismos de suspensión y los valores previamente calculados. Los datos obtenidos muestran que el prototipo 1, al tener un chasis más cercano al suelo en comparación con otros prototipos que están diseñados con una altura superior centrados en superar escaleras (Kim et al., 2012), mantiene una gran estabilidad y mejor eficiencia al momento de aumentar la velocidad. Algunos estudios están enfocados en el control y seguimiento del prototipo en terrenos blandos, mientras que el presente estudio se caracteriza por diseñar una superficie irregular, observando que el conjunto del prototipo adquiere mayor movilidad y agilidad al sortear obstáculos de diferente nivel (Chen et al., 2021). La altura de los obstáculos que permite cruzar el prototipo está dentro de 1.5 a 2.7 veces la altura de sus ruedas, esta varía de acuerdo al centro de masa del Rover.

Tabla 1. Valores comparativos entre prototipos

\begin{tabular}{|c|c|c|c|c|}
\hline Nombre & Unidades & Calculados & Prototipo 1 & Prototipo 2 \\
\hline Fuerza & $\mathrm{N}$ & 94 & 94 & 94 \\
\hline Altura máxima & $\mathrm{cm}$ & 26 y 51.96 & 32.68 & 70 \\
\hline Longitud máxima & $\mathrm{cm}$ & 60 & 66.73 & 74.34 \\
\hline Velocidad máxima & $m S$ & 0.25 & 1.2 & 1.13 \\
\hline Aceleración máxima & $m s 2$ & 0.02 & 0.096 & 0.031 \\
\hline Ángulo máximo & Grados $^{\circ}$ & 30 & 37.83 & 39.86 \\
\hline
\end{tabular}

La tabla 2 muestra la comparativa del factor de seguridad de cada uno de los eslabones de los dos prototipos, los valores obtenidos a través de la simulación estática garantizan la seguridad y fiabilidad. El diseño de los eslabones Rocker-Bogie representa una gran fiabilidad y seguridad para cualquier tipo de industria que requiera realizar una tarea específica sobre una superficie irregular. Comparado a otros prototipos, este brinda un factor de seguridad superior 
incluso a los cálculos establecidos previamente. Por lo cual, el diseño es óptimo y adecuado (Seralathan et al., 2020).

Tabla 2. Análisis F.S.

\begin{tabular}{|c|c|c|c|}
\hline Nombre & Detalle & F.S Calculado & F.S Simulado \\
\hline \multirow{2}{*}{ Rocker } & Prototipo 1 & \multirow{2}{*}{} & 2.01 \\
\cline { 2 - 2 } & Prototipo 2 & \multirow{2}{*}{1.45} & 3.83 \\
\cline { 2 - 2 } Bogie & Prototipo 1 & & 12 \\
\cline { 2 - 2 } & Prototipo 2 & & 12.1 \\
\hline
\end{tabular}

\section{Conclusiones y recomendaciones}

En esta investigación se ha optado por el diseño de dos prototipos. Los dos de tipo Rocker-Bogie, pero con un enfoque diferente. El uno tiene el chasis más pegado a la superficie para una mayor estabilidad, mientras que el otro tiene una mayor altura para sortear obstáculos de mayor nivel. Según los resultados obtenidos, el prototipo 1 es el que mejor se desempeña a lo largo de la pista de prueba al tener mayor fuerza de agarre y empuje para superar los obstáculos gracias a la estabilidad que representa tener el chasis pegado a la superficie. La altura de los obstáculos que permite cruzar el prototipo está dentro de 1.5 a 2.7 veces la altura de sus ruedas, no obstante, el diseño está sujeto al área de aplicación, pudiendo variar dicha altura. Se puede aplicar en áreas como la agrícola, minera e industrial en las que se requiera realizar una tarea especifica sobre una superficie irregular.

La distancia mínima y máxima alcanzada por el prototipo 1 al atravesar la pista es de 66.73 $\mathrm{cm}$ la mayor longitud medida de centro a centro, entre la rueda frontal y la posterior del Rover. La velocidad máxima alcanzada es de $1.1 \mathrm{~m} / \mathrm{s}$ y la mínima es de $-0.3 \mathrm{~m} / \mathrm{s}$. La fuerza de tracción facilita que el Rover sea capaz de superar los obstáculos que se encuentran a lo largo de su camino, siendo capaz de adquirir un torque promedio de $36.78 \mathrm{~N}^{*} \mathrm{~m}$. Mientras que con el segundo prototipo la distancia mayor adquirida por los ejes de las ruedas frontal y posterior es de $74.34 \mathrm{~cm}$ y la menor de $60 \mathrm{~cm}$. La altura máxima alcanzada es de 70cm con respecto a la llanta ubicada en el punto más bajo disponible, conservando su posición de equilibrio adecuado. La velocidad máxima alcanzada en secciones sin obstáculos es de $113 \mathrm{~cm} / \mathrm{s}$, este valor representa momentos en los cuales una o algunas de las ruedas tienen un contacto mínimo con el suelo.

Se recomienda utilizar un software programable para establecer el ángulo de giro que deben emplearse en los servos motores Nema, que es controlado con una tarjeta LITEBEE que permite mantener sub rutinas. Así mismo, el tablero se integra con PX4FMU + PX4IO.Pixhawk está con la nueva tecnología de chips y sensores de 32 bits que complementan el funcionamiento adecuado de la implementación de sensores junto al mecanismo desarrollado, evitando que este pueda chocar o volcarse en terrenos que contengan gran cantidad de imperfecciones.

Es importante considerar la aplicación del prototipo, de ello dependerá el enfoque del diseño cinemático basado en grados de libertad. Algunas superficies requerirán que se aumente el número de ruedas para adaptarse a la superficie. Además, se recomienda optar por un método de control simultáneo de seguimiento para rastrear la trayectoria deseada y mantener pequeñas fuerzas internas, utilizando la información de movimiento en paralelo con la información de fuerza. 
Para este estudio, los eslabones Rocker-Bogie junto con el chasis son de material de aluminio 1060, las llantas y neumáticos material ABS, parámetros necesarios para la validación estática. Para futuras líneas de investigación se recomienda utilizar distintos tipos de material que aligeren la estructura y tengan mayor agarre a la superficie, además de agregar variadores de velocidad capaces de incrementar o reducir rápidamente según la demanda, con el fin de optimizar el traslado del Rover. También se puede incurrir en acondicionar sensores ultrasónicos de precisión para un seguimiento controlado de la trayectoria deseada mediante la implementación de un sistema IA.

\section{Referencias}

Bogue, R. (2012). Robots for Space Exploration. Industrial Robot: An International Journal, 39(4): 323.328. doi:10.1108/01439911211227872

Cabrera, J.; Jurado, F., y Vera, D. (2021). Simulación de un deshidratador híbrido indirecto activo mediante el software ANSYS. Enfoque UTE, 12(4): 29-44. https://doi.org/10.29019/enfoqueute.771

Chen, C., et al. (2021). Simultaneous Control of Trajectory Tracking and Coordinated Allocation of RockerBogie Planetary Rovers. Mechanical Systems and Signal Processing, 151: 107312. https://doi. org/https://doi.org/10.1016/j.ymssp.2020.107312

Chinchkar, D., et al. (2017). Design of Rocker Bogie Mechanism. International Advanced Research Journal in Science, Engineering and Technology, 4(1): 46-50.

Conte, R. (2013). Robot Espaciales. Recuperado de http://haciaelespacio.aem.gob.mx/revistadigital/articul.php?interior $=39$

Eisen, H. J., et al. (1998). Sojourner Mars Rover Thermal Performance. SAE Transactions, 107: 697-707. http://www.jstor.org/stable/44735797

Elishakoff, I. (2017). Probabilistic Methods in the Theory of Structures: Strength of Materials, Random Vibrations, and Random Buckling. Mechanical Systems and Signal Processing (151): 107312

Gonzalez, R., y lagnemma, K. (2017). Slippage Estimation and Compensation for Planetary Exploration Rovers. State of the Art and Future Challenges. Journal of Field Robotics, 35(4): 564577. doi:10.1002/rob.21761

Gul, F., et al. (2021). Multi-Robot Space Exploration: An Augmented Arithmetic Approach. IEEE Access, (9): 107738-107750. doi:10.1109/ACCESS.2021.3101210.

$\mathrm{Hu}$, J., et al. (2020). Voronoi-Based Multi-Robot Autonomous Exploration in Unknown Environments via Deep Reinforcement Learning. IEEE Transactions on Vehicular Technology, 12 (69): 1441314423. doi:10.1109/TVT.2020.3034800

Hu, Y., et al. (2019). Design and Validation of a Self-Driven Joint Model for Articulated Arm Coordinate Measuring Machines. Applied Sciences, 9(15). https://doi.org/10.3390/app9153151

Karras, J. T., et al. (2017). Pop-up Mars Rover with Textile-enhanced Rigid-flex PCB body. IEEE International Conference on Robotics and Automation (ICRA): 5459-5466. https://doi.org/10.1109/ ICRA.2017.7989642

Kim, D., et al. (2012). Optimal Design and Kinetic Analysis of a Stair-climbing Mobile Robot with RockerBogie Mechanism. Mechanism and Machine Theory, 50: 90-108. https://doi.org/https://doi. org/10.1016/j.mechmachtheory.2011.11.013

Kornfeld, R. et al. (2014). Verification and Validation of the Mars Science Laboratory/Curiosity Rover Entry, Descent, and Landing System. Journal of Spacecraft and Rockets, 51(4): 1251-1269. https://doi. org/10.2514/1.A32680

Meghdari, A., et al. (2005). A Novel Approach for Optimal Design of a Rover Mechanism. Journal of Intelligent and Robotic Systems, 44(4): 291-312. https://doi.org/10.1007/s10846-005-9013-5. 
Misha, S., et al. (2020). Simulation CFD and Experimental Investigation of PVT Water System Under Natural Malaysian Weather Conditions. Energy Re-ports, 6: 28-44. https://doi.org/https://doi. org/10.1016/j.egyr.2019.11.162

Musto, J. (2010). The Safety Factor: Case Studies in Engineering Judgment. International Journal of Mechanical Engineering Education, 38(4): 286-296. https://doi.org/10.7227/IJMEE.38.4.2

Sanguino, T. (2017). 50 Years of Rovers for Planetary Exploration: A Retrospective Review for Future Directions. Robotics and Autonomous Systems, 94: 172-185. https://doi.org/https://doi. org/10.1016/j.robot.2017.04.020

Seralathan, S., et al. (2020). Static Structural Analysis of Wheel Chair Using a Rocker Bogie Mechanism. Materials Today: Proceedings, 33: 3583-3590. https://doi.org/https://doi.org/10.1016/j.matpr.2020.05.658

Smith, B., Y Saaj, C. (2009). Biologically Inspired Nano-rovers: Innovative and Low-Cost Technologies Using Shape Memory Alloys. In Proceedings of 60th International Astronautical Congress.

Tashtoush, T. et al. (2020). Reverse-Twister Swarm Search Algorithm Design: NASA Swarmathon Competition. International Journal of Research Studies in Computer Science and Engineering, 1 (7): 29-36. https://doi.org/10.20431/2349-4859.0701004

Toupet, O., et al. (2018). Traction Control Design and Integration Onboard the Mars Science Laboratory Curiosity Rover. IEEE Aerospace Conference: 1-20. https://doi.org/10.1109/AER0.2018.8396761

Tunstel, E. (2007). Prototype Rover Field Testing and Planetary Surface Operations. Proceedings of the 2007 Workshop on Performance Metrics for Intelligent Systems, 196-203. https://doi. org/10.1145/1660877.1660905

Volpe, R., et al. (1996). The Rocky 7 Mars rover prototype. Proceedings of IEEE/RSJ International Conference on Intelligent Robots and Systems. IROS '96, 3: 1558-1564. https://doi.org/10.1109/ IROS.1996.569020

Xu, Y., y Wang, Z. (2021). Visual Sensing Technologies in Robotic Welding: Recent Research Developments and Future Interests. Sensors and Actuators. A Physical, 320: 112551. https://doi.org/10.1016/j. sna.2021.112551

Yang, J.; Dong, M., y Ye, J. (2017). A Literature Review of the Rocker-Bogie Suspension for the Planetary Rover. AISR, 150: 137-142

Zheng, J., et al. (2018). Design and Terramechanics Analysis of a Mars Rover Utilizing Active Suspension. Mechanism and Machine Theory, 128: 125-149. https://doi.org/https://doi.org/10.1016/j.mechmachtheory.2018.05.002 